\title{
Patterns of brain oscillations across different electrode montages in transcranial pulsed current stimulation Alejandra C. Vasquez ${ }^{\mathrm{a}}$, Aurore Thibaut ${ }^{\mathrm{a}, \mathrm{b}}$, Leon Morales-Quezada ${ }^{\mathrm{a}}$, Jorge Leite ${ }^{\mathrm{a}, \mathrm{c}}$ and Felipe Fregni ${ }^{\mathrm{a}}$
}

\begin{abstract}
Transcranial pulsed current stimulation (tPCS) is a neuromodulatory technique that has been studied in the last decade. Several parameters have been assessed independently to optimize the effects. Our aim was to explore the effects of tPCS using different montages on cortical brain oscillations indexed by power spectrum and interhemispheric coherence in different electroencephalography frequency bands. Twenty healthy individuals were randomized to receive either active IPCS or sham intervention using the following bilateral montages: ear clip (conventional), ear hook, or mastoid placement. Electroencephalography was recorded before and after the electroencephalography intervention to assess tPCS-induced after effects. Our results showed that active tPCS with bimastoid montage increased significantly alpha absolute power $(P=0.0166)$ and low alpha $(P=0.0014)$ in the frontal region, as well as in the low alpha power spectrum in the central $(P=0.0001)$ and parieto-occipital regions $(P=0.0068)$ compared with the other montages. For interhemispheric coherence analysis, the Kruskal-Wallis test showed a significant main effect of group for theta
\end{abstract}

$(P=0.0012)$ in the frontal region, mainly for ear-clip montage.
Our findings evidenced that tPCS delivered through different electrode montages exert different effects on cortical brain oscillations and thus have a different neural signature. We discuss the implications of these findings as well as potential clinical explorations of this technique. NeuroReport 00:000-000 Copyright (c) 2017 Wolters Kluwer Health, Inc. All rights reserved.

NeuroReport 2017, 00:000-000

Keywords: electroencephalography, electrode montage, interhemispheric coherence, noninvasive brain stimulation, plasticity, power spectrum, transcranial pulsed current stimulation

aSpaulding Neuromodulation Center, Harvard Medical School, Spaulding Rehabilitation Hospital, Boston, Massachusetts, USA, 'Department of Neurology, Coma Science Group, GIGA-Research, University of Liege, Liege, Belgium and ${ }^{\mathrm{c} N e u r o p s y c h o p h y s i o l o g y ~ L a b o r a t o r y, ~ C I P s i, ~ S c h o o l ~ o f ~ P s y c h o l o g y, ~ U n i v e r s i t y ~ o f ~}$ Minho, Braga, Portugal

Correspondence to Felipe Fregni, MD, PhD, MPH, 79/96, 13th Street Charlestown, MA 02129, USA

Tel: + 1617573 2499; fax: +1617952 6153; e-mail: felipe.fregni@ppcr.hms.harvard.edu

Received 30 January 2017 accepted 2 March 2017

finite element head model, and was used to evaluate the cortical electrical field, its distribution, and peak intensities. The authors evaluated different electrode configurations, including in-ear and over-ear montages [10], and showed that in addition to the effect on cortical areas, tPCS could reach subcortical structures. In this study, all montages were positioned within the margins of the ears and even relatively minor changes within these electrodes induced different electrical fields. Interestingly, the authors showed that the ear-hook montage, resembling the headphones' positioning, produced the highest cortical field, especially in the temporal cortex [10].

On the basis of this previous study [10], here, we aimed to evaluate the effects of tPCS using different montages on cortical brain oscillations in healthy individuals indexed by high-resolution quantitative electroencephalography (EEG) changes for power and interhemispheric coherence.

\section{Participants and methods Study design}

This was a randomized, double-blind, and shamcontrolled study. We used a computer-generated block randomization list with blocks of four. The sham
One computational modeling study [10] assessed variations in electrode positioning with tPCS using MRI-derived 
condition was also randomized for the different electrode montages. The study was approved by the local institutional review board (IRB) and carried out according to the Declaration of Helsinki [11]. Written informed consent was obtained from the participants before their inclusion in the study.

\section{Participants}

The eligibility criteria were as follows: healthy volunteers between 18 and 65 years of age with no history of unstable medical illness or neurologic/psychiatric conditions. In addition, the presence of traumatic brain injury with loss of consciousness, history of drug or alcohol abuse, history of brain surgery or presence of metallic implants, current pregnancy, and previous exposure to tPCS were ruled out.

\section{Intervention}

tPCS was administered using a custom-made investigational, battery-powered current stimulator. It delivers stimulation through biphasic square wave pulses; the pulse width is randomly generated and varies between 1 and $20 \mathrm{~ms}$. The participants received a single session of tPCS with a peak pulse amplitude of $2 \mathrm{~mA}$ and a frequency that ranged between 6 and $10 \mathrm{~Hz}$ (additional details of stimulation are shown in the study by Vasquez et al. [12]). Participants were randomized into four groups to receive active tPCS or sham through the following montages: (a) ear clip, (b) ear hook, (c) bimastoid, and (d) sham. The positioning of the electrodes was selected on the basis of computational studies [10]. For ear-clip and ear-hook montages, each electrode consisted of a circular metallic plate of $\sim 0.785 \mathrm{~cm}^{2}$ covered with a cotton felt, placed in the inferior lobule of each ear for ear clip, and positioned in the scaphoid fossa underneath the superior part of the ear's helix for the ear-hook montage.

The bimastoid montage used the same alternating bilateral pattern as the previous montages, but used $3.2 \mathrm{~cm}$ circular electrodes with a multistick Gel (Axelgaard Manufacturing Co. Ltd, Fallbrook, California, USA) placed bilaterally over the mastoid process.

The participants received $20 \mathrm{~min}$ of $\mathrm{tPCS}$ according to their allocation group. For the sham condition, the device was turned off automatically $30 \mathrm{~s}$ after its onset.

At the end of the stimulation, each participant completed an Adverse Events questionnaire.

\section{Electroencephalographic recording}

The EEG was recorded using a 32-channel EEG system (Enobio 32; Neuroelectrics, Barcelona, Spain). The signal was sampled at $500 \mathrm{~Hz}$ and filtered using a bandpass of 0.1-35 Hz. EEG was recorded 6 min with the eyes open and $6 \mathrm{~min}$ with the eyes closed before and after the stimulation.

\section{Power and interhemispheric coherence analysis}

EEGLab [13] and MATLAB (MATLAB R2012a, 2000;

The MathWorks Inc., Natick, Massachusetts, USA) were used to process and analyze the EEG data. Data were high-pass filtered at $1 \mathrm{~Hz}$ and low-pass filtered at $35 \mathrm{~Hz}$, with subsequent removal of amplitudes higher than 200 $\mu \mathrm{V}$ as well as artifact components by both computational algorithms and manual inspection. Data were transformed into frequency domains using Fast Fourier transformation with $5 \mathrm{~s}$ epochs and an overlap of $50 \%$ to calculate the power $\left(\mu \mathrm{V}^{2}\right)$ of the following bandwidths: theta $(4-8 \mathrm{~Hz})$, alpha $(8-13 \mathrm{~Hz})$, low alpha $(8-10 \mathrm{~Hz})$, and high alpha $(10-13 \mathrm{~Hz})$. The channels were averaged by bandwidth for frontal, central, parietal, temporal, and occipital brain regions. We excluded F3 and F4 because of important artifacts.

We calculated the interhemispheric coherence for the same bands across the electrode pairs. Welch's averaged modified periodogram method was used to calculate the coherence of signal $x$ and $y$, representing each electrode site.

\section{Behavioral tasks}

We used the Mini-Mental State Examination [14], the Stroop color word test (E-Prime 2.0 software; Psychology Software Tools, Pittsburgh, Pennsylvania, USA) [15], and the word list memory task [16] to assess any detrimental effects of tPCS on cognition. They were performed in the above-mentioned order immediately before the EEG preceding the stimulation and after the second EEG recording.

\section{Statistical analysis}

Statistical analysis was carried out using STATA 14.1 (Stata Corporation, College Station, Texas, USA). The artifact-free, eyes-closed EEG data were tested for normality using the Shapiro-Wilk test $(w=0.000)$. For baseline characteristics, continuous variables were compared using one-way analysis of variance and categorical variables were analyzed using a $\chi^{2}$-test. To analyze the cognitive assessments, mixed-model analysis of variances were performed with time as the within-subject factor (two levels: pre vs. post) and stimulation condition as the between-subject factor (with four levels).

The Kruskal-Wallis test was used to compare the effects of stimulation on power and coherence variables. The Mann-Whitney test was used for post-hoc comparisons. The dependent variables for EEG power and coherence in each bandwidth were calculated as the difference between poststimulation and prestimulation. The independent variable for power and coherence analysis was Group. Statistical significance was set at 0.05.

\section{Results}

Twenty participants were enrolled in the study, of whom 19 completed the study (mean age: $25.7 \pm 4.04,11$ women). One participant dropped out due to discomfort during the 
stimulation (ear-hook montage). The participants' demographic characteristics were similar between the four groups (age: $P=0.275$, sex: $P=0.585$, ethnicity: $P=0.629$ ).

\section{Electroencephalography power spectrum analysis - group effects}

Bimastoid montage was first compared against ear-clip (conventional) montage for power and we found that bimastoid montage induced a significant increase within the low alpha bandwidth over the frontal $(P=0.0042)$ and central regions $(P=0.0053)$. Then, compared with the sham, we also found a significant increase in power in low alpha but not only over the frontal $(P=0.0311)$ and central $(P=0.0001)$ regions, but also over the parietooccipital area $(P=0.0128)$ (Table 1$)$.

When ear clip and ear hook were compared with sham, an increase in theta power was observed over parietooccipital areas $(P=0.0095$ and 0.001 , respectively). On comparing the two techniques, we found that ear-clip montage induced a more significant increase in power within the theta bandwidth over the central region, than ear-hook montage $(P=0.006)$.

These results suggest that bimastoid montage significantly increases the absolute power of alpha bandwidth compared with other montages (Fig. 1). We then carried out a secondary analysis to assess the differences between bimastoid and ear-clip montages. For the bimastoid montage, there was a significant increase in alpha power $(P=0.0166)$ and low alpha $(P=0.0014)$ in the frontal region, as well as in low alpha power spectrum in the central $(P=0.0001)$ and parieto-occipital regions $(P=0.0068)$. There were no significant results for the alpha band with the ear-clip montage.

\section{Interhemispheric coherence}

The Kruskal-Wallis test showed a significant main effect of group for theta (0.0012) in the frontal region. Post-hoc analysis evidenced significant differences when ear clip was compared with the rest of the montages. Ear-clip montage induced a higher interhemispheric theta coherence ('Table 1).

\section{Cognitive assessments}

There were nonsignificant values between the four groups in changes (pre-postintervention) for all three cognitive tests: Mini-Mental State Examination $(P=0.413)$, Stroop task $(P=0.405)$, and the word list memory task $(P=0.425)$.

\section{Discussion}

Our findings included: (a) bimastoid tPCS can enhance mainly the power spectrum of the alpha bandwidth in the frontocentral and parieto-occipital brain regions compared with the other montages and (b) for the ear-clip montage, we observed an increase in theta interhemispheric coherence for the frontal area, supporting previous studies [1-3]. These findings suggest that a single session of tPCS has a consistent impact on cortical activity, as measured by EEG-derived connectivity, for the alpha domain and that specific electrophysiological parameters can be elicited by particular electrode montages, making tPCS a technique in which top-down (high internal processing) and bottom-up (low internal demands) processes could be targeted independently.

Our study provides insight into the role of electrode montage in tPCS neurophysiological effects. As shown in the Datta et al.'s [10] modeling study, even small changes in electrode montage can alter the current flow patterns in the brain. Our results on healthy participants, by means of EEG measures, support these assumptions as

Table 1 Kruskal-Wallis and related post-hoc (Mann-Whitney) analysis for electroencephalography power and interhemispheric coherence among groups

\begin{tabular}{|c|c|c|c|c|c|}
\hline & \multicolumn{2}{|c|}{ Kruskal-Wallis } & \multicolumn{3}{|c|}{ Mann-Whitney } \\
\hline & $P$-value & $\chi^{2}($ d.f. $)$ & Intervention & $P$-value & $Z$-score \\
\hline \multicolumn{6}{|l|}{ Power } \\
\hline \multicolumn{6}{|l|}{ Frontal } \\
\hline \multirow[t]{3}{*}{ Low alpha } & 0.0132 & 10.735 (3) & Ear clip - bimastoid & 0.0042 & -2.86 \\
\hline & & & Ear hook - bimastoid & 0.0064 & -2.725 \\
\hline & & & Bimastoid - sham & 0.0311 & 2.155 \\
\hline \multicolumn{6}{|l|}{ Central } \\
\hline \multirow[t]{3}{*}{ Low alpha } & 0.0001 & 20.927 (3) & Ear clip - bimastoid & 0.0053 & -2.79 \\
\hline & & & Ear hook - bimastoid & 0.0005 & -3.456 \\
\hline & & & Bimastoid - sham & 0.0001 & 3.952 \\
\hline \multirow[t]{2}{*}{ Theta } & 0.0105 & 11.248 (3) & Ear clip - ear hook & 0.006 & 2.745 \\
\hline \multirow{2}{*}{\multicolumn{6}{|c|}{ Parieto-occipital }} \\
\hline & & & & & \\
\hline \multirow[t]{2}{*}{ Low alpha } & 0.0143 & 10.568 (3) & Ear hook - bimastoid & 0.0037 & -2.899 \\
\hline & & & Bimastoid - sham & 0.0128 & 2.49 \\
\hline \multirow[t]{2}{*}{ Theta } & 0.0046 & 13.029 (3) & Ear clip - sham & 0.001 & 3.296 \\
\hline & & & Ear hook - sham & 0.0095 & 2.595 \\
\hline \multicolumn{6}{|c|}{ Interhemispheric coherence } \\
\hline \multirow[t]{3}{*}{ Theta } & 0.0012 & $11.103(3)$ & Ear clip - ear hook & 0.0047 & 2.83 \\
\hline & & & Ear clip - bimastoid & 0.0087 & 2.625 \\
\hline & & & Ear clip - sham & 0.0107 & 2.551 \\
\hline
\end{tabular}


Fig. 1

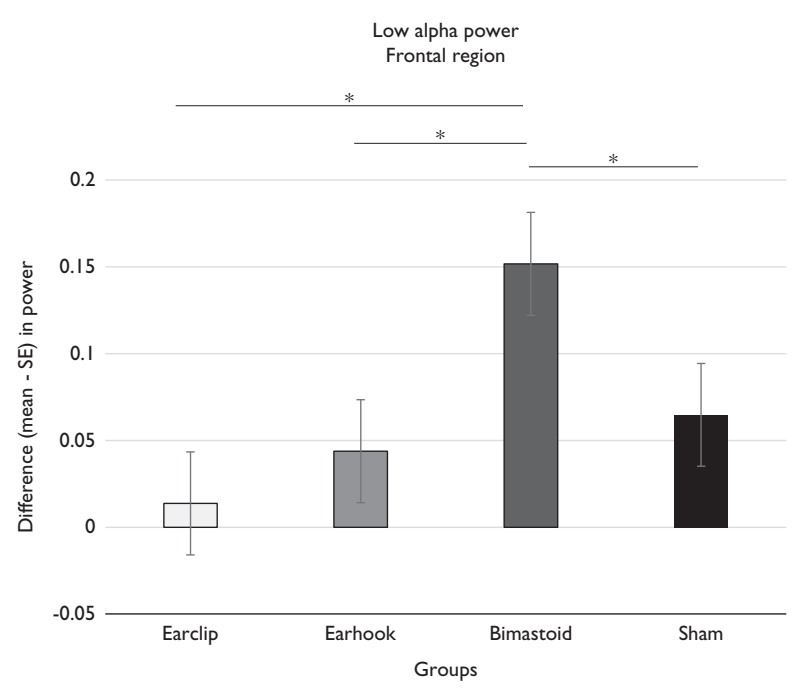

Low alpha power. Difference postminus preintervention (mean $-\mathrm{SE})$ in power for low alpha bandwidth in the frontal area $-\mu \mathrm{V} 2 .{ }^{\star} P<0.05$.

earlobes and bimastoid montages induced distinct neurophysiological changes. The importance of electrode location has been shown for other NIBS techniques, such as tDCS [17], as well as in studies using modeling with clinical correlates [9].

A probable theoretical explanation for the main effect observed in the power spectrum with the bimastoid montage could be the spatial distribution of this synchronous process. The direct excitation of the neurons under the area of the electrodes may induce variations of membrane dynamics that can further activate distant neuronal networks in the cortex [18]. This cortico-cortical communication is reflected in the increase in alpha and low alpha power for different cortical brain areas despite the initial input site. In addition, this may further support that alpha can also be explained by cortico-cortical interactions, rather than only the thalamo-cortical circuit $[19,20]$.

For the tPCS bimastoid montage, similar electrode positioning and results have been reported for other NIBS such as transcranial alternating current stimulation (tACS). This technique introduces a weak sinusoidal electrical current, which, similar to tPCS, is capable of directly modulating the ongoing brain oscillations. A study evaluating the effect of tACS showed an increase in the individual alpha frequency range when applied in the parieto-occipital region [21]. Another study using transorbital tACS at individual alpha frequency reported a significant increase of the EEG alpha power at different scalp locations, such as occipital regions [20]. This cortico-cortical connectivity observed with tACS has been further supported by an MRI study, with the increase in brain activity not only under the area of the electrodes but in more distant brain regions [22].
These aforementioned results are similar to our findings which lead us to consider that bimastoid tPCS can have a similar effect to tACS. Indeed, it is well known that neurons within the central thalamus have extensive anatomical connectivity with the forebrain [23]. Thus, it would be possible to hypothesize that our findings rely on the activation of the cortico-thalamo-cortical loop through the stimulation of posterior areas of the brain, followed by the connection between the thalamus and the prefrontal cortex (through the striatopallidal modulatory system), leading to an increased activity over the frontal cortex. This implies that bimastoid tPCS modulates the top-down control of neuronal synchronization and thus connectivity.

Our second observation of the increase in theta interhemispheric coherence in the frontal region for the earclip montage implies a second hypothesis: tPCS seems to influence first theta coherence or have a stronger effect on coherence than power. As shown in head modeling studies, tPCS seems to influence subcortical brain structures, such as the brainstem or the thalamus [10]. Through the activation of the brainstem, tPCS may exert an effect on the reticular formation and, consequently, on the modulation of signals arriving to the thalamus. On the basis of the current and previous studies with tPCS, it seems feasible that the subthalamic nuclei and thalamus, in their role as electrographical generators (via thalamocortical circuits), could stimulate the bottom-up connectivity and exert an indirect effect on cortical excitability, translated by the increase in frontal interhemispheric theta coherence following tPCS.

On the basis of this, it can be hypothesized that bimastoid tPCS may have similar mechanisms to tACS and thus stimulate the top-down connectivity, whereas the ear-clip tPCS montage would have more effects on bottom-up connectivity. Knowing which network to stimulate has a marked impact on the clinical effect of tCS. For instance, in pathologies related to disruptions in the thalamo-cortical connectivity, such as schizophrenia [24] and autism [25], ear-clip tPCS could be preferred, whereas for conditions involving cortical dysfunction, specifically with reduced alpha rhythm such as neuropathic pain [6], bimastoid tPCS might be more efficient. The similarity to tACS mechanisms also widens the field of exploration between these two techniques. Future studies should compare the neurophysiologic effects of both techniques, a probable synergistic effect, and potential therapeutic applications.

\section{Acknowledgements}

The authors thank Alejandra Malavera for her initial contribution to the project.

This study was supported in part by a grant from the Labuschagne Foundation. 


\section{Conflicts of interest}

There are no conflicts of interest.

\section{References}

1 Castillo Saavedra L, Morales-Quezada L, Doruk D, Rozinsky J, Coutinho L, Faria $P$, et al. QEEG indexed frontal connectivity effects of transcranial pulsed current stimulation (tPCS): a sham-controlled mechanistic trial. Neurosci Lett 2014; 577:61-65.

2 Morales-Quezada L, Castillo-Saavedra L, Cosmo C, Doruk D, Sharaf I, Malavera A, Fregni F. Optimal random frequency range in transcranial pulsed current stimulation indexed by quantitative electroencephalography. Neuroreport 2015; 26:747-752.

3 Morales-Quezada L, Saavedra LC, Rozisky J, Hadlington L, Fregni F. Intensity-dependent effects of transcranial pulsed current stimulation on interhemispheric connectivity: a high-resolution qEEG, sham-controlled study. Neuroreport 2014; 25:1054-1058.

4 Morales-Quezada L, Cosmo C, Carvalho S, Leite J, Castillo-Saavedra L, Rozisky JR, Fregni F. Cognitive effects and autonomic responses to transcranial pulsed current stimulation. Exp brain Res 2015; 233:701-709.

5 Güntekin B, Saatçi E, Yener G. Decrease of evoked delta, theta and alpha coherences in Alzheimer patients during a visual oddball paradigm. Brain Res 2008; 1235:109-116.

6 Meneses FM, Queirós FC, Montoya P, Miranda JGV, Dubois-Mendes SM, Sá $\mathrm{KN}$, et al. Patients with rheumatoid arthritis and chronic pain display enhanced alpha power density at rest. Front Hum Neurosci 2016; 10:395.

7 Saturnino GB, Antunes A, Thielscher A. On the importance of electrode parameters for shaping electric field patterns generated by tDCS. Neuroimage 2015; 120:25-35.

8 DaSilva AF, Volz MS, Bikson M, Fregni F. Electrode positioning and montage in transcranial direct current stimulation. J Vis Exp 2011; 51:2744.

9 Mendonca ME, Santana MB, Baptista AF, Datta A, Bikson M, Fregni F Araujo CP. Transcranial DC stimulation in fibromyalgia: optimized cortical target supported by high-resolution computational models. J Pain 2011; 12:610-617.

10 Datta A, Dmochowski JP, Guleyupoglu B, Bikson M, Fregni F. Cranial electrotherapy stimulation and transcranial pulsed current stimulation: a computer based high-resolution modeling study. Neuroimage 2013; 65: 280-287.

11 [No authors listed]. Declaration of Helsinki. Recommendations guiding doctors in clinical research. Adopted by the World Medical Association in 1964. Wis Med J 1967; 66:25-26.
12 Vasquez A, Malavera A, Doruk D, Morales-Quezada L, Carvalho S, Leite J, Fregni $F$. Duration dependent effects of transcranial pulsed current stimulation (tPCS) indexed by electroencephalography. Neuromodulation 2016; 19:679-688.

13 Delorme A, Makeig S. EEGLAB: an open source toolbox for analysis of single-trial EEG dynamics including independent component analysis. J Neurosci Methods 2004; 134:9-21.

14 Tombaugh Tom N, Mclntyre NJ. The Mini-Mental State Examination: a comprehensive review. J Am Geriatr Soc 1992; 40:922-935.

15 Comalli PE, Wapner S, Werner H. Interference effects of stroop color-word test in childhood, adulthood, and aging. J Genet Psychol 1962; 100:47-53.

16 Welsh KA, Butters N, Mohs RC, Beekly D, Edland S, Fillenbaum G, Heyman A. The Consortium to Establish a Registry for Alzheimer's Disease (CERAD). Part V. A normative study of the neuropsychological battery. Neurology 1994; 44:609-614.

17 Nitsche Ma, Nitsche Ma, Paulus W, Paulus W. Excitability changes induced in the human motor cortex by weak transcranial direct current stimulation. J Physiol 2000; 527 (Pt 3):633-639.

18 Roland PE, Hilgetag CC, Deco G. Cortico-cortical communication dynamics. Front Syst Neurosci 2014; 8:19.

19 Lopes da Silva F. Neural mechanisms underlying brain waves: from neural membranes to networks. Electroencephalogr Clin Neurophysiol 1991; 79:81-93.

20 Merlet I, Birot G, Salvador R, Molaee-Ardekani B, Mekonnen A, Soria-Frish A et al. From oscillatory transcranial current stimulation to scalp EEG changes: a biophysical and physiological modeling study. PLoS One 2013; 8:e57330.

21 Vossen A, Gross J, Thut G. Alpha power increase after transcranial alternating current stimulation at alpha frequency (a-tACS) reflects plastic changes rather than entrainment. Brain Stimul 2015; 8:499-508.

22 Cabral-Calderin Y, Williams KA, Opitz A, Dechent P, Wilke M. Transcranial alternating current stimulation modulates spontaneous low frequency fluctuations as measured with fMRI. Neuroimage 2016; 141:88-107.

23 Van der Werf YD, Witter MP, Groenewegen HJ. The intralaminar and midline nuclei of the thalamus. Anatomical and functional evidence for participation in processes of arousal and awareness. Brain Res Rev 2002; 39:107-140.

24 Anticevic A, Cole MW, Repovs G, Murray JD, Brumbaugh MS, Winkler AM, et al. Characterizing thalamo-cortical disturbances in schizophrenia and bipolar illness. Cereb Cortex 2014; 24:3116-3130.

25 Nair A, Treiber JM, Shukla DK, Shih P, Müller RA. Impaired thalamocortical connectivity in autism spectrum disorder: a study of functional and anatomical connectivity. Brain 2013; 136:1942-1955. 